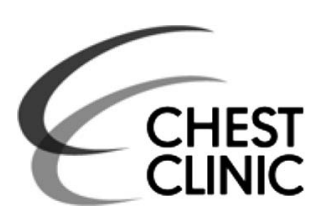

University Children's Hospita at Dr. von Haunersches Kinderspital, LMU Munich, Munich, Germany

\section{Correspondence to} Cihan Papan, University Children's Hospital at Dr. von Haunersches Kinderspital, LMU Munich, Lindwurmstr. 4, 80337 Munich, Germany; cihan.papan@med. uni-muenchen.de

Received 20 September 2016 Revised 24 October 2016 Accepted 30 October 2016 Published Online First 21 November 2016

\title{
Pott's disease: a major issue for an unaccompanied refugee minor
}

\author{
Cihan Papan, Ulrich von Both, Matthias Kappler, Birgit Kammer, Matthias Griese, \\ Johannes Huebner
}

\section{ABSTRACT}

The incidence of TB in children in Germany has been on a rise since 2008, especially among foreign-born individuals. With rapidly increasing numbers of refugees from the numerous areas of conflict, this increase in incidence is not expected to halt, neither in Germany nor in Europe in general. We report a case of insufficient tracking in a 16-year-old unaccompanied refugee minor from Somalia who had a positive interferon $\gamma$ release assay on arrival in Germany. No actions were undertaken, until 6 months later, an X-ray showed prominent hilar enlargement. Nine months later, the patient presented to our hospital with abdominal pain, vomiting and B symptoms. Workup revealed a paravertebral abscess due to Pott's disease, a skeletal manifestation of Mycobacterium tuberculosis disease. The patient made a full recovery after a combination therapy for a total of 9 months.

\section{CASE REPORT}

A 16-year-old adolescent presented to our tertiary care centre in March 2014, with a 2-week history of abdominal pain, vomiting, fever, night sweats and weight loss. He had fled alone from Somalia to Germany 15 months earlier. Routine screening on arrival had revealed chronic hepatitis $\mathrm{B}$ and a positive interferon $\gamma$ release assay (IGRA, QuantiFERON-TB Gold). No further actions were taken until 6 months later, when a chest radiograph was ordered, showing a prominent right hilum. Again, no further action was taken.

On admission at our hospital 9 months later, epigastric tenderness was noted, while lungs were clear to auscultation. Laboratory tests yielded elevated inflammatory markers (ie, $\mathrm{C}$ reactive protein $107 \mathrm{mg} / \mathrm{L}$, erythrocyte sedimentation rate $35 \mathrm{~mm} /$ hours). Chest radiography showed a mediastinal mass and hilar enlargement. CT and MRI of the spine showed a paravertebral mass consistent with an abscess (figure 1A, B), several intravertebral lesions suspicious for spondylitis (figure 2) and enlarged lymph nodes.

A confirmatory second IGRA yielded a positive result. In a CT-guided drainage of the abscess, acidfast bacilli were detected by microscopy, identified as Mycobacterium tuberculosis (MTB) by PCR and subsequently confirmed by culture. Of note, repeated sputum microscopy yielded no acid-fast bacilli, but MTB culture eventually revealed a positive result. Drug sensitivity testing showed a fully sensitive organism.
The patient responded well to a standard fourdrug antituberculous regimen consisting of isoniazid (INH), rifampicin (RMP), pyrazinamide (PZA) and ethambutol (EMB). After 3 months, PZA and EMB were stopped. INH and RMP were continued for a total of 9 months. At follow-up 1 year after discharge, the patient was in a good condition with full weight regain. Spinal MRI now showed complete resolution of the abscess, but fatty remodelling of multiple vertebrae.

Skeletal TB is an extrapulmonary manifestation accounting for $2.5 \%-5 \%$ of $\mathrm{TB}$ cases in the European Union. The most common form is TB of the spine (Pott's disease) accounting for about half of all cases. ${ }^{1}$ During the initial bacteriaemic phase, the bacilli disseminate to the bones, where they lie dormant until postprimary disease. Main risk factors for skeletal TB are HIV and living in a highprevalence area. Diagnosis is dependent on a high grade of suspicion, imaging and biopsy of the affected site. If untreated, Pott's disease can lead to severe deformations and long-term neurological sequelae.

The incidence of TB in Germany has reached a plateau since 2008. On the other hand, incidence of childhood TB, which is a surrogate for ongoing transmission in the adult population, has shown a mild increase. ${ }^{2}$ The proportion of foreign-born individuals with $\mathrm{TB}$ is also on a rise, while the percentage among native Germans is falling. In parallel, the number of unaccompanied refugee minors (URMs) taken into custody by the youth welfare has more than tripled since 2009 (3015 children)

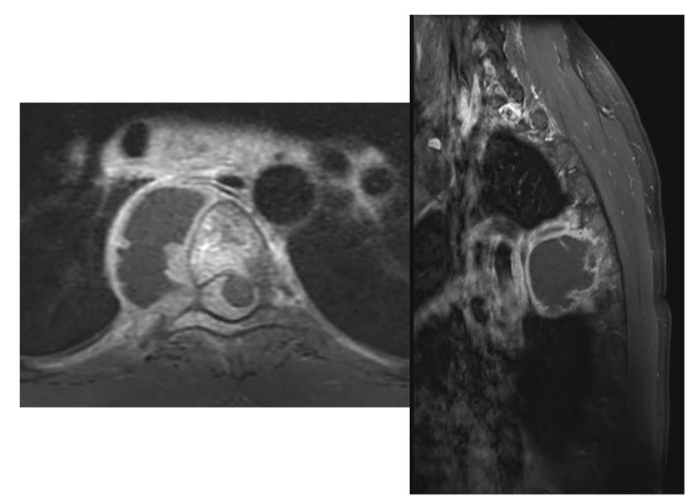

Figure 1 Contrast-enhanced T1-weighted images of the thoracic spine in axial (A) and sagittal (B) orientation reveal a paravertebral abscess on the right side. Note signal enhancement of the vertebral body, rib, soft tissues, meninges and mediastinal lymph nodes. 


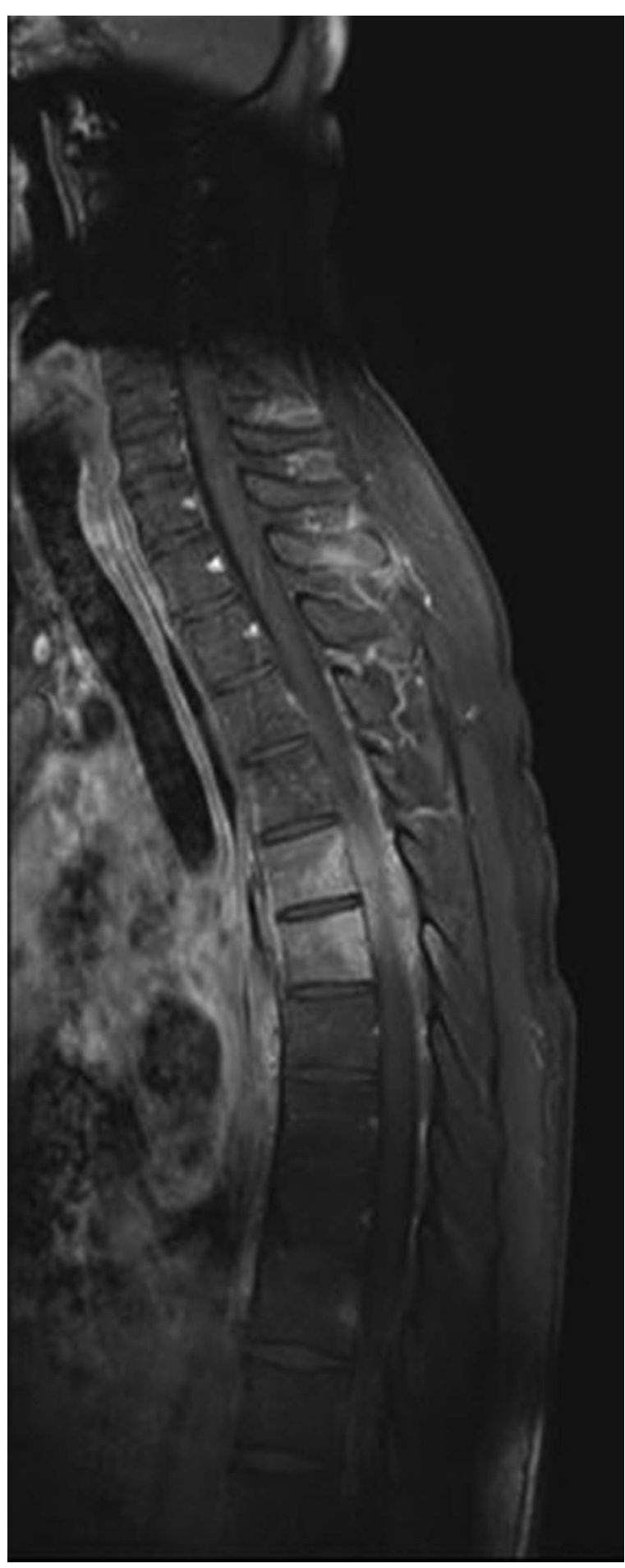

Figure 2 MRI of thoracic spine in sagittal orientation; contrast-enhanced T1-weighted image depicts signal enhancement of the vertebra extending to the meninges and anterior soft tissues. according to the German Federal Association for URMs, who reported 10404 custodies in 2014 . $^{3}$

Since September 2015, Germany and Munich in particular has been facing an unprecedented wave of refugees including more URM, reflecting an ever-growing number of worldwide conflicts and displaced people. The first 2 weeks of September 2015 alone have seen about 60000 refugees arriving at Munich central station, a substantial amount of these consisting of URM. $^{4}$ As of August 2016, estimates amount to 1.1 million refugees having arrived in Germany in 2015 with an additional 300000 in $2016 .^{5}$

Beyond the well-established health risks inherent to migration, URMs are a particularly vulnerable group. On arrival, they are taken into custody by the youth welfare office, before being appointed to a guardian who is then responsible for the enforcement of the child's rights. While native German children have their parents who, if needed, can proactively request pending results and ask for follow-up, URM are lacking such a tight safety net.

While we debate modalities of TB screening, ${ }^{6}$ insufficient tracking of screening findings has emerged as a major issue. ${ }^{7}$ This situation proved calamitous in our patient, who fully recovered from his infection. Screening of refugees without an adequate follow-up is unacceptable. Not only medical awareness of 'forgotten diseases' has to be raised but also political solutions are urgently needed to live up to our humanitarian obligation and help those who are most in need of help.

Acknowledgements We thank the patient and his legal guardian for participation.

Contributors CP took care of the patient, performed literature search and drafted the initial manuscript; UvB reviewed and revised the manuscript; MK, MG and $\mathrm{JH}$ took care of the patient, reviewed and revised the manuscript; BK made the radiological diagnosis, reviewed and revised the manuscript; all authors approved the final manuscript as submitted and agree to be accountable for all aspects of the work. Written consent to publication was obtained.

\section{Competing interests None.}

Patient consent Obtained.

Provenance and peer review Not commissioned; externally peer reviewed.

\section{REFERENCES}

1 Pigrau-Serrallach C, Rodriguez-Pardo D. Bone and joint tuberculosis. Eur Spine J 2013;22(Suppl 4):556-6.

2 RKI-Bericht zur Epidemiologie der Tuberkulose in Deutschland für 2013 [RKI report on the epidemiology of tuberculosis in Germany 2013], 2014. http://www.rki.de/DE/ Content/InfAZ/T/Tuberkulose/Archiv Berichte TB in Dtl tab.html

3 Das Bundesamt in Zahlen 2014-Asyl [The Federal Office in figures 2014-asylum], 2015. http://www.bamf.de/SharedDocs/Anlagen/DE/Publikationen/Broschueren/ bundesamt-in-zahlen-2014.html?nn=1694460

4 Nicolai T, Fuchs 0, von Mutius E. Caring for the wave of refugees in Munich. N Engl J Med 2015;373:1593-5.

5 Germany expects up to 300,000 refugees in 2016, official says 2016. http://www. theguardian.com/world/2016/aug/28/germany-300000-refugees-2016-bamf

6 van der Werf MJ, Lönnroth K. Pre-entry, post-entry, or no tuberculosis screening? Lancet Infect Dis 2014;14:1171-2.

7 Harstad I, Jacobsen GW. Poor follow-up after screening for tuberculosis. Tidsskr Nor Laegeforen 2012;132:14-16. 